\title{
On the largest dynamic monopolies of graphs with a given average threshold
}

\author{
Kaveh Khoshkhah Manouchehr Zaker* \\ Department of Mathematics, \\ Institute for Advanced Studies in Basic Sciences, \\ Zanjan 45137-66731, Iran
}

\begin{abstract}
Let $G$ be a graph and $\tau$ be an assignment of nonnegative integer thresholds to the vertices of $G$. A subset of vertices $D$ is said to be a $\tau$-dynamic monopoly, if $V(G)$ can be partitioned into subsets $D_{0}, D_{1}, \ldots, D_{k}$ such that $D_{0}=D$ and for any $i \in\{0, \ldots, k-1\}$, each vertex $v$ in $D_{i+1}$ has at least $\tau(v)$ neighbors in $D_{0} \cup \ldots \cup D_{i}$. Denote the size of smallest $\tau$-dynamic monopoly by $d y n_{\tau}(G)$ and the average of thresholds in $\tau$ by $\bar{\tau}$. We show that the values of $d y n_{\tau}(G)$ over all assignments $\tau$ with the same average threshold is a continuous set of integers. For any positive number $t$, denote the maximum $d y n_{\tau}(G)$ taken over

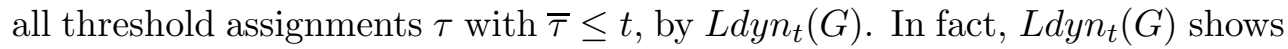
the worst-case value of a dynamic monopoly when the average threshold is a given number $t$. We investigate under what conditions on $t$, there exists an upper bound for $\operatorname{Ldyn}_{t}(G)$ of the form $c|G|$, where $c<1$. Next, we show that $\operatorname{Ldyn}_{t}(G)$ is coNP-hard for planar graphs but has polynomial-time solution for forests.
\end{abstract}

Mathematics Subject Classification: 05C69, 05C85, 91D30

Keywords: Spread of influence in graphs; Irreversible dynamic monopolies

\section{Introduction}

In this paper we deal with simple undirected graphs. For any such graph $G=(V, E)$, we denote the cardinality of its vertex set by $|G|$ and the edge density of graph $G$ by

*E-mail: mzaker@iasbs.ac.ir 
$\epsilon(G):=|E| /|G|$. We denote the degree of a vertex $v$ in $G$ by $\operatorname{deg}_{G}(v)$. For other graph theoretical notations we refer the reader to [2]. By a threshold assignment for the vertices of $G$ we mean any function $\tau: V(G) \rightarrow \mathbb{N} \cup\{0\}$. A subset of vertices $D$ is said to be a $\tau$-dynamic monopoly of $G$ or simply $\tau$-dynamo of $G$, if for some nonnegative integer $k$, the vertices of $G$ can be partitioned into subsets $D_{0}, D_{1}, \ldots, D_{k}$ such that $D_{0}=D$ and for any $i, 1 \leq i \leq k$, the set $D_{i}$ consists of all vertices $v$ which has at least $\tau(v)$ neighbors in $D_{0} \cup \ldots \cup D_{i-1}$. Denote the smallest size of any $\tau$-dynamo of $G$ by $d y n_{\tau}(G)$. Dynamic monopolies are in fact modeling the spread of influence in social networks. The spread of innovation or a new product in a community, spread of opinion in Yes-No elections, spread of virus in the internet, spread of disease in a population are some examples of these phenomena. Obviously, if for a vertex $v$ we have $\tau(v)=\operatorname{deg}_{G}(v)+1$ then $v$ should belong to any dynamic monopoly of $(G, \tau)$. We call such a vertex $v$ self-opinioned (from another interpretation it can be called vaccinated vertex). Irreversible dynamic monopolies and the equivalent concepts target set selection and conversion sets have been the subject of active research in recent years by many authors $[3,4,6,7,8,10,11,12,13]$.

In this paper by $(G, \tau)$ we mean a graph $G$ and a threshold assignment for the vertices of $G$. The average threshold of $\tau$, denoted by $\bar{\tau}$, is $\sum_{v \in V(G)} \tau(v) /|G|$. In Proposition 1 we show that the values of $d y n_{\tau}(G)$ over all threshold assignments with the same average threshold form a continuous set of integers. The maximum element of this set has been studied first time in [10], where the following notation was introduced. Let $t$ be a non-negative rational number such that $t|G|$ is an integer, then $\operatorname{Dyn}_{t}(G)$ is defined as $D y n_{t}(G)=\max _{\tau: \bar{\tau}=t} d y n_{\tau}(G)$. The smallest size of dynamic monopolies with a given average threshold was introduced and studied in [13. Dynamic monopolies with given average threshold was also recently studied in [5]. In the definition of $\operatorname{Dyn}_{t}(G)$, it is assumed that $t|G|$ is integer. In order to consider all values of $t$, we modify a little bit the definition. But we are forced to make a new notation, i.e. $\operatorname{Ldyn}_{t}(G)$ (which stands for the largest dynamo). The formal definition is as follows.

Definition 1. Let $G$ be a graph and $t$ a positive number. We define $\operatorname{Ldyn}_{t}(G)=$ $\max \left\{\operatorname{dyn}_{\tau}(G) \mid \bar{\tau} \leq t\right\}$. Assume that a subset $D \subseteq V(G)$ and an assignment of thresholds $\tau_{0}$ are such that $\overline{\tau_{0}} \leq t,|D|=\operatorname{dyn}_{\tau_{0}}(G)=\operatorname{Ldyn}_{t}(G)$ and $D$ is a $\tau_{0^{-}}$ dynamic monopoly of $\left(G, \tau_{0}\right)$. Then we say $\left(D, \tau_{0}\right)$ is a $t$-Ldynamo of $G$.

$\operatorname{Ldyn}_{t}(G)$ does in fact show the worst-case value of a dynamic monopoly when the average threshold is a prescribed given number. The following concept is motivated by the concept of dynamo-unbounded family of graphs, defined in [12] concerning the smallest size of dynamic monopolies in graphs.

Definition 2. Let for any $n \in \mathbb{N}, G_{n}$ be a graph and $t_{n}$ be a number such that $0 \leq t_{n} \leq 2 \epsilon\left(G_{n}\right)$. We say $\left\{\left(G_{n}, t_{n}\right)\right\}_{n \in \mathbb{N}}$ is Ldynamo-bounded if there exists a constant $\lambda<1$ such that for any $n, \operatorname{Ldyn}_{t_{n}}\left(G_{n}\right) \leq \lambda\left|G_{n}\right|$. 
Outline of the paper is as follows. In Section 2, we show that the values of $\operatorname{dyn}_{\tau}(G)$ over all assignments $\tau$ with the same average threshold is a continuous set of integers (Proposition 1). Then we obtain a necessary and sufficient condition for a family of graphs to be Ldynamo-bounded (Propositions 3 and 4). In Section 3, it is shown that the decision problem Ldynamo $(k)$ (to be defined later) is coNP-hard for planar graphs (Theorem 1) but has polynomial-time solution for forests (Theorem 3).

\section{Some results on $\operatorname{Ldyn}_{t}(G)$}

We first show that the values of $\operatorname{dyn}_{\tau}(G)$ over all threshold assignments $\tau$ with the same average threshold are continuous. We need the following lemma from [11.

Lemma 1. 11] Let $G$ be a graph and $\tau$ and $\tau^{\prime}$ be two threshold assignments to the vertices of $G$ such that $\tau(u)=\tau^{\prime}(u)$ for all vertices $u$ of $G$ except for exactly one vertex, say $v$. Then

$$
\begin{cases}d y n_{\tau}(G)-1 \leq d y n_{\tau^{\prime}}(G) \leq d y n_{\tau}(G), & \text { if } \tau(v)>\tau^{\prime}(v), \\ d y n_{\tau}(G) \leq d y n_{\tau^{\prime}}(G) \leq d y n_{\tau}(G)+1, & \text { if } \tau(v)<\tau^{\prime}(v)\end{cases}
$$

The continuity result is as follows.

Proposition 1. Let $\tau$ and $\tau^{\prime}$ be two threshold assignments for the vertices of $G$ such that $\bar{\tau}=\bar{\tau}^{\prime}$. Let also $r$ be an integer such that dyn $n_{\tau}(G) \leq r \leq d y n_{\tau^{\prime}}(G)$. Then there exists $\tau^{\prime \prime}$ with $\bar{\tau}=\bar{\tau}^{\prime \prime}$ such that dyn ${ }_{\tau^{\prime \prime}}(G)=r$.

Proof. For any two threshold assignments $\tau$ and $\tau^{\prime}$ with the same average threshold, define $\delta\left(\tau, \tau^{\prime}\right)=\sum_{v: \tau(v)>\tau^{\prime}(v)}\left(\tau(v)-\tau^{\prime}(v)\right)$. We prove the proposition by the induction on $\delta\left(\tau, \tau^{\prime}\right)$. If $\delta\left(\tau, \tau^{\prime}\right)=0$ then for any vertex $v, \tau(v) \leq \tau^{\prime}(v)$. But the average thresholds are the same, hence $\tau=\tau^{\prime}$ and the assertion is trivial. Let $k \geq 1$ and assume that the proposition holds for any two $\tau$ and $\tau^{\prime}$ with the same average threshold such that $\delta\left(\tau, \tau^{\prime}\right) \leq k$. We prove it for $k+1$. Assume that $\tau$ and $\tau^{\prime}$ are given such that $\delta\left(\tau, \tau^{\prime}\right)=k+1$ and $\tau \neq \tau^{\prime}$. Define $W=\left\{v: \tau(v)>\tau^{\prime}(v)\right\}$. Let $w \in W$. There exists a vertex $u$ such that $\tau(u)<\tau^{\prime}(u)$. Since otherwise by $\bar{\tau}=\bar{\tau}^{\prime}$ we would have $\tau=\tau^{\prime}$. Define a new threshold $\tau^{\prime \prime}$ as follows. For any vertex $v$ with $v \notin\{u, w\}$ set $\tau^{\prime \prime}(v)=\tau(v)$. Set also $\tau^{\prime \prime}(w)=\tau(w)-1$ and $\tau^{\prime \prime}(u)=\tau(u)+1$. We have $\delta\left(\tau^{\prime \prime}, \tau^{\prime}\right) \leq k$, also the average threshold of $\tau^{\prime \prime}$ is the same as that of $\tau$. So the assertion holds for $\tau^{\prime \prime}$ and $\tau^{\prime}$. By Lemma 1 we have $\left|d y n_{\tau}(G)-d y n_{\tau^{\prime \prime}}(G)\right| \leq 1$. We conclude that the assertion holds for $\tau$ and $\tau^{\prime}$ too.

Let $G$ be a graph and $t$ be a positive number such that $t|G|$ is integer. Let $\tau$ be any assignment with average $t$ such that $\tau(v) \leq \operatorname{deg}_{G}(v)$ for any vertex $v$. Let $d_{1} \leq d_{2} \leq$ 
$\ldots \leq d_{n}$ be a degree sequence of $G$ in increasing form. It was proved in [10] that the size of any $\tau$-dynamic monopoly of $G$ is at $\operatorname{most} \max \left\{k: \sum_{i=1}^{k}\left(d_{i}+1\right) \leq n t\right\}$. The proof of this result in [10] shows that if we allow $\tau(v)=\operatorname{deg}_{G}(v)+1$ for some vertices $v$ of $G$, then the same assertion still holds. We have the following proposition concerning this fact.

Proposition 2. Let $t$ be a positive number. Assume that in the definition of $\operatorname{Ldyn}_{t}(G)$, the threshold assignments are allowed to have self-opinioned vertices. Then Ldyn $n_{t}(G)$ can be easily obtained by a polynomial-time algorithm.

Proof. Let $d_{1} \leq d_{2} \leq \ldots \leq d_{n}$ be a degree sequence of $G$ in increasing form. By the argument we made before Proposition 2, we have $\operatorname{Ldyn}_{t}(G) \leq \max \{k$ : $\left.\sum_{i=1}^{k}\left(d_{i}+1\right) \leq n t\right\}$. Let $k_{0}=\max \left\{k: \sum_{i=1}^{k}\left(d_{i}+1\right) \leq n t\right\}$. We obtain a threshold assignment $\tau$ as follows.

$$
\tau\left(v_{i}\right)= \begin{cases}\operatorname{deg}_{G}\left(v_{i}\right)+1 & i \leq k_{0} \\ 0 & \text { otherwise }\end{cases}
$$

Let $D=\left\{v_{1}, v_{2}, \ldots, v_{k_{0}}\right\}$. It's clear that $(D, \tau)$ is a $t$-Ldynamo of $G$.

In [10, it was proved that there exists an infinite sequence of graphs $G_{1}, G_{2}, \ldots$ such that $\left|G_{n}\right| \rightarrow \infty$ and $\lim _{n \rightarrow \infty} L d y n_{\epsilon\left(G_{n}\right)}\left(G_{n}\right) /\left|G_{n}\right|=1$. In the following, we show that a stronger result holds. In fact we show that not only the same result holds for $\operatorname{Ldyn}_{k \epsilon\left(G_{n}\right)}\left(G_{n}\right)$, where $k$ is any constant with $0<k \leq 2$, but also it holds for any sequence $k_{n}$ for which $k_{n}\left|G_{n}\right| \rightarrow \infty$. In opposite direction, Proposition 4 shows that if $k_{n}=\mathcal{O}\left(1 /\left|G_{n}\right|\right)$ then $\lim _{n \rightarrow \infty} \operatorname{Ldyn}_{k_{n} \epsilon\left(G_{n}\right)}\left(G_{n}\right) /\left|G_{n}\right| \neq 1$.

Proposition 3. There exists an infinite sequence of graphs $\left\{\left(G_{n}, \tau_{n}\right)\right\}_{n=1}^{\infty}$ satisfying $\left|G_{n}\right| \rightarrow \infty$ and $\epsilon\left(G_{n}\right) /\left|G_{n}\right|=o\left(\bar{\tau}_{n}\right)$ such that

$$
\lim _{n \rightarrow \infty} \frac{\operatorname{Ldyn}_{\bar{\tau}}\left(G_{n}\right)}{\left|G_{n}\right|}=1 .
$$

Proof. We construct $G_{n}$ as follows. The vertex set of $G_{n}$ is disjoint union of a complete graph $K_{n}$ and $n$ copies of complete graphs $K_{n+1}$. There exists exactly one edge between each copy of $K_{n+1}$ and $K_{n}$. Set $\tau_{n}(v)=0$ for each vertex $v$ in $K_{n}$ and $\tau_{n}(v)=\operatorname{deg}(v)$ for each vertex $v$ in any copy of $K_{n+1}$. It is clear that any dynamic monopoly of $G_{n}$ includes at least $n$ vertices of each copy of $K_{n+1}$ and hence $\operatorname{Ldyn}_{\bar{\tau}}\left(G_{n}\right) \geq n^{2}$. Then we have

$$
1 \geq \lim _{n \rightarrow \infty} \frac{\operatorname{Ldyn}_{\bar{\tau}}\left(G_{n}\right)}{\left|G_{n}\right|} \geq \lim _{n \rightarrow \infty} \frac{n^{2}}{n(n+2)}=\lim _{n \rightarrow \infty} \frac{n}{n+2}=1 .
$$


To complete the proof we show that $\frac{\bar{\tau}_{n}}{\left|E\left(G_{n}\right)\right| /\left|V\left(G_{n}\right)\right|^{2}} \rightarrow \infty$.

$$
\lim _{n \rightarrow \infty} \frac{\bar{\tau}_{n}}{\left|E\left(G_{n}\right)\right| /\left|V\left(G_{n}\right)\right|^{2}}=\lim _{n \rightarrow \infty} \frac{\left(n^{2}+n+1\right) /(n+2)}{\left(n^{2}+n+n\left(n+n^{2}\right)\right) / 2\left(n^{2}+2 n\right)^{2}}=\infty
$$

Proposition 3 shows that if $t_{n}$ is such that $\epsilon\left(G_{n}\right) /\left|G_{n}\right|=o\left(t_{n}\right)$ then $\left\{\left(G_{n}, t_{n}\right)\right\}_{n}$ is not necessarily Ldynamo-bounded. In opposite direction, the next proposition shows that if there exists a positive number $c$ such that $t_{n}$ satisfies $t_{n} \leq c \epsilon\left(G_{n}\right) /\left|G_{n}\right|$, then any family $\left\{\left(G_{n}, t_{n}\right)\right\}_{n}$ is Ldynamo-bounded.

Proposition 4. Let $G$ be a graph and $c$ and $t$ be two constants such that $t \leq c \frac{\epsilon(G)}{|G|}$. Then

$$
\operatorname{Ldyn}_{t}(G)<\frac{c}{c+1}|G|
$$

Proof. Let $n$ be the order of $G$. If $n<c / 2$, then $\lceil c n /(c+1)\rceil=n$ and hence the inequality $\operatorname{Ldyn}_{t}(G)<c|G| /(c+1)$ is trivial. Assume now that $n \geq c / 2$. Let $d_{1} \leq d_{2} \leq \ldots \leq d_{n}$ be a degree sequence of $G$ in increasing form and set $k_{0}=$ $\max \left\{k: \sum_{i=1}^{k}\left(d_{i}+1\right) \leq n t\right\}$. As we mentioned before, by a result from [10] we have $\operatorname{Ldyn}_{t}(G) \leq k_{0}$. The assumption $t \leq c(\epsilon(G) / n)$ implies $n t \leq(c / 2 n) \sum_{i=1}^{n} d_{i}$ and hence $\sum_{i=1}^{k_{0}}\left(d_{i}+1\right) \leq(c / 2 n) \sum_{i=1}^{n} d_{i}$ or equivalently $(2 n / c) \leq\left(\sum_{i=1}^{n} d_{i}\right) / \sum_{i=1}^{k_{0}}\left(d_{i}+\right.$ 1). Assume on the contrary that $k_{0} \geq c n /(c+1)$. Then

$$
\frac{2 n}{c} \leq \frac{\sum_{i=1}^{k_{0}} d_{i}+\sum_{i=k_{0}+1}^{n} d_{i}}{\left(\sum_{i=1}^{k_{0}} d_{i}\right)+\frac{c}{c+1} n} \leq \frac{\left(\sum_{i=1}^{k_{0}} d_{i}\right)+\frac{n^{2}}{c+1}}{\left(\sum_{i=1}^{k_{0}} d_{i}\right)+\frac{c}{c+1} n}
$$

Therefore

$$
\frac{2 n-c}{c} \sum_{i=1}^{k_{0}} d_{i} \leq \frac{n^{2}}{c+1}-\frac{2 n^{2}}{c+1} .
$$

The left hand side of the last inequality is positive but the other side is negative. This contradiction implies $k_{0}<c n /(c+1)$, as required.

\section{Algorithmic results}

Algorithmic results concerning determining $d y n_{\tau}(G)$, with various types of threshold assignments such as constant thresholds or majority thresholds, were studied in 
[4, 6, 7]. In this section, we first show that it is a coNP-hard problem on planar graphs to compute the size of $D$ such that $(D, \tau)$ is a $k \epsilon(G)$-Ldynamo of $G$. Then we prove that the same problem has a polynomial-time solution for forests. The formal definition of the decision problem concerning Ldynamo is the following, where $k$ is any arbitrary but fixed real number with $0<k \leq 2$.

\section{Name: LARGEST DYNAMIC MONOPOLY (Ldynamo(k))}

Instance: A graph $G$ on say $n$ vertices and a positive integer $d$.

Question: Is there an assignment of thresholds $\tau$ to the vertices of $G$ with $n \bar{\tau}=$ $\lfloor n k \epsilon(G)\rfloor$ such that $d y n_{\tau}(G) \geq d$ ?

The following theorem shows coNP-hardness of the above problem. Recall that Vertex Cover (VC) asks for the smallest number of vertices $S$ in a graph $G$ such that $S$ covers any edge of $G$. Denote the smallest cardinality of any vertex cover of $G$ by $\beta(G)$. The problem VC is NP-complete for planar graphs [9].

Theorem 1. For any fixed $k$, where $0<k \leq 2, \operatorname{Ldynamo}(k)$ is coNP-hard even for planar graphs.

Proof. We make a polynomial time reduction from VC (planar) to our problem. Let $\langle G, l\rangle$ be an instance of $\mathrm{VC}$, where $G$ is planar. Define $s=4|E(G)| \times$ $\max \{1,1 / k\}+14$ and set $p=\lfloor(k s-2) /(2-k)\rfloor-|E(G)|$. Construct a graph $H$ from $G$ as follows. To each vertex $v$ of $G$ attach a star graph $K_{1, s-1}$ in such a way that $v$ is connected to the central vertex of the star graph. Consider one of these star graphs and let $y$ be a vertex of degree one in it. Add a path $P$ of length $p-1$ starting from $y$ (see Figure 1). The path $P$ intersects the rest of the graph only in $y$. Call the resulting graph $H$. Since $G$ is planar, $H$ is planar too.

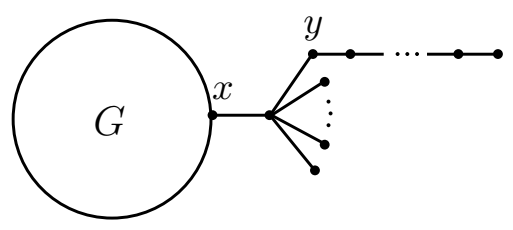

Figure 1: graph $H$

We claim that $\langle G, l\rangle$ is a yes-instance of $\mathrm{VC}$ if and only if $\langle H, l+\lfloor p / 2\rfloor+1\rangle$ is a no-instance of $\operatorname{Ldynamo}(k)$. From the construction of $H$, we have $|E(H)|=$ $|E(G)|+s+p$. Then since $p=\lfloor(k s-2) /(2-k)\rfloor-|E(G)|$ we have

$$
\begin{aligned}
& p \leq(k s-2) /(2-k)-|E(G)| \\
\Rightarrow & 2 p+2|E(G)|+2 \leq k(s+p+|E(G)|) \\
\Rightarrow & 2 p+2|E(G)|+2 \leq\lfloor k|E(H)|\rfloor .
\end{aligned}
$$


Also from the value of $p$ we have

$$
\begin{aligned}
& p \geq(k s-2) /(2-k)-|E(G)|-1 \\
\Rightarrow & 2 p+2|E(G)|+2+(2-k)>k(s+p+|E(G)|) \\
\Rightarrow & 2 p+2|E(G)|+2+\lfloor 2-k\rfloor \geq\lfloor k|E(H)|\rfloor \\
\Rightarrow & 2 p+2|E(G)|+3 \geq\lfloor k|E(H)|\rfloor .
\end{aligned}
$$

Assume first that $\langle G, l\rangle$ is a no-instance of VC. Then $\beta(G) \geq l+1$. We construct a threshold assignment $\tau$ for graph $H$ as follows.

$$
\tau(v)= \begin{cases}\operatorname{deg}_{H}(v) & v \in G \cup P, \\ 0 & \text { otherwise. }\end{cases}
$$

It is easily seen that $\bar{\tau} \leq k \epsilon(H)$ and also $d y n_{\tau}(H)=\beta(G)+\lfloor p / 2\rfloor$. Therefore $<H, l+\lfloor p / 2\rfloor+1>$ is a yes-instance for $\operatorname{Ldynamo}(k)$.

Let $\langle G, l\rangle$ be a yes-instance of VC. Then $\beta(G)<l+1$. Assume that $(D, \tau)$ is a $(k \epsilon(H))$-Ldynamo of $H$. The assumption $s>4|E(G)|+14$ implies $|D \cap(H \backslash G)| \leq$ $\lfloor p / 2\rfloor$. From the other hand, $|D \cap G| \leq \beta(G)<l+1$. Hence $|D|<l+\lfloor p / 2\rfloor+1$. This shows that $<H, l+\lfloor p / 2\rfloor+1>$ is a no-instance for $\operatorname{Ldynamo}(k)$. This completes the proof.

In the rest of this section we obtain a polynomial-time solution for forests (Theorem 3). We need some prerequisites. We will make use of the concept of resistant subgraphs, defined in [12] as follows. Given $(G, \tau)$, any induced subgraph $K \subseteq G$ is said to be a $\tau$-resistant subgraph in $G$, if for for any vertex $v \in K$ the inequality $\operatorname{deg}_{K}(v) \geq \operatorname{deg}_{G}(v)-\tau(v)+1$ holds, where $\operatorname{deg}_{G}(v)$ is the degree of $v$ in $G$. The following proposition in [12] shows the relation between resistant subgraphs and dynamic monopolies.

Proposition 5.([12]) A set $D \subseteq G$ is a $\tau$-dynamo of graph $G$ if and only if $G \backslash D$ does not contain any resistant subgraph.

The following lemma provides more information on resistant subgraphs which are also triangle-free.

Lemma 2. Assume that $(G, \tau)$ is given. Let also $H$ be a triangle-free $\tau$-resistant subgraph in $G$ and $e=u v$ be any arbitrary edge with $u, v \in H$. Let $\tau^{\prime}$ be defined as follows

$$
\tau^{\prime}(w)= \begin{cases}\tau(w) & \text { if } w \notin H, \\ 0 & \text { if } w \in H \backslash\left\{u, v^{\prime}\right\} \\ \operatorname{deg}_{G}(v) & \text { if } w=v \\ \operatorname{deg}_{G}(u) & \text { if } w=u\end{cases}
$$


Then $\overline{\tau^{\prime}} \leq \bar{\tau}$.

Proof. Since $H$ is triangle-free, then $|H| \geq \operatorname{deg}_{H}(u)+\operatorname{deg}_{H}(v)$. From the definition of the resistant subgraphs, for any vertex $w \in H$, one has $\tau(w) \geq \operatorname{deg}_{G \backslash H}(w)+1$. Hence the following inequalities hold.

$$
\begin{aligned}
\sum_{w \in H} \tau(w) & \geq \sum_{w \in H}\left(\operatorname{deg}_{G \backslash H}(w)+1\right) \\
& \geq|H|+\operatorname{deg}_{G \backslash H}(u)+\operatorname{deg}_{G \backslash H}(v) \\
& \geq \operatorname{deg}_{H}(u)+\operatorname{deg}_{H}(v)+\operatorname{deg}_{G \backslash H}(u)+\operatorname{deg}_{G \backslash H}(v) \\
& =\operatorname{deg}_{G}(u)+\operatorname{deg}_{G}(v) .
\end{aligned}
$$

It turns out that $\sum_{w \in G} \tau^{\prime}(w) \leq \sum_{w \in G} \tau(w)$ and hence $\overline{\tau^{\prime}} \leq \bar{\tau}$.

By a (zero,degree)-assignment we mean any threshold assignment $\tau$ for the vertices of a graph $G$ such that for each vertex $v \in V(G)$, either $\tau(v)=0$ or $\tau(v)=\operatorname{deg}_{G}(v)$. The following remark is useful and easy to prove. We omit its proof.

Remark 1. Assume that $(G, \tau)$ is given where $\tau$ is (zero, degree)-assignment. Let $G_{1}$ be the subgraph of $G$ induced on $\left\{v \in G \mid \tau(v)=\operatorname{deg}_{G}(v)\right\}$. Then every minimum vertex cover of $G_{1}$ is a minimum $\tau$-dynamo of $G$, and vice versa.

The following theorem concerning (zero,degree)-assignments in forests is essential in obtaining an algorithm for $t$-Ldynamo of forests for a given $t$.

Theorem 2. Let $F$ be a forest and $t$ be a positive constant. There exists a (zero,degree)assignment $\tau^{\prime}$ such that $\overline{\tau^{\prime}} \leq t$ and

$$
\operatorname{Ldyn}_{t}(F)=d y n_{\tau^{\prime}}(F) .
$$

Proof. Let $(D, \tau)$ be a $t$-Ldynamo of $F$. We prove the theorem by induction on $|D|$. Assume first that $|D|=1$. Then by Proposition 5, $F$ has at least one $\tau$-resistant subgraph say, $F^{\prime}$. Let $u$ and $v$ be two adjacent vertices in $F^{\prime}$. Let $\tau^{\prime}$ be the threshold assignment constructed in Lemma 2 $\operatorname{such}$ that $\tau^{\prime}(u)=\operatorname{deg}_{F}(u)$ and $\tau^{\prime}(v)=\operatorname{deg}_{F}(v)$. Modify $\tau^{\prime}$ so that $\tau^{\prime}(w)=0$ for every vertex $w \in F \backslash\{u, v\}$. It is clear that $\tau^{\prime}$ is a (zero,degree)-assignment. The edge $u v$ is a $\tau^{\prime}$-resistant subgraph in $F$ and hence $d y n_{\tau}(F)=\operatorname{Ldyn}_{t}(F)=1$. This proves the induction assertion in this case.

Now assume that the assertion holds for any forest $F$ with $|D|<k$. Let $F$ be a forest with $\operatorname{Ldyn}_{t}(F)=k$ and $D$ be a $t$-Ldynamo of $F$ with $|D|=k$. Let also $F_{1}$ be the largest $\tau$-resistant subgraph of $F$. For any $v \in F_{1}$, set $\varphi(v)=\tau(v)-\operatorname{deg}_{F \backslash F_{1}}(v)$. By the definition of resistant subgraphs, $\varphi(v)>0$. It is clear that $\operatorname{dyn}_{\varphi}\left(F_{1}\right)=k$. 
We show that there exists a (zero,degree)-assignment $\tau_{1}^{\prime}$ for $F_{1}$ such that $\left(D_{1}, \tau_{1}^{\prime}\right)$ is a $\bar{\varphi}$-Ldynamo of $F_{1}$ with $\left|D_{1}\right|=k$.

Let $T$ be a connected component of $F_{1}$. Consider $T$ as a top-down tree, where the toppest vertex is considered as the root of $T$. Since $T$ is a $\varphi$-resistant subgraph in $F_{1}$, it implies that $D_{1} \cap T$ is not the empty set. We argue that $D_{1}$ can be chosen in such a way that it does not contain any vertex $w \in T$ with $\varphi(w)=1$, except possibly the root. The reason is that if $w \in D_{1} \cap T$ with $\varphi(w)=1$, then we replace $w$ by its nearest ancestor (with respect to the root of $T$ ) whose threshold is not 1 ; and if there is no such ancestor then we replace $w$ by the root. Let $v \in D_{1} \cap T$ be the farthest vertex from the root of $T$. Let $T_{v}$ be the subtree of $T$ consisting of $v$ and its descendants. Obviously $T_{v} \cap D_{1}=\{v\}$.

Now we show that $T_{v}$ is a $\varphi$-resistant subgraph in $F_{1}$. For each vertex $w \in T_{v} \backslash\{v\}$, since $\varphi(w) \geq 1$ and $\operatorname{deg}_{F_{1} \backslash T_{v}}(w)=0$, then $\varphi(w) \geq \operatorname{deg}_{F_{1} \backslash T_{v}}(w)+1$. We have also $\varphi(v) \geq \operatorname{deg}_{F_{1} \backslash T_{v}}(v)+1$. Since if $\varphi(v)=1$, then $v$ is the root of $T$ and $T_{v}=T$ and hence $\operatorname{deg}_{F_{1} \backslash T_{v}}(v)=0$. And if $\varphi(v)>1$, then $\operatorname{deg}_{F_{1} \backslash T_{v}}(v) \leq 1$. This proves that $T_{v}$ is a $\varphi$-resistant subgraph in $F_{1}$. Let $v^{\prime}$ be an arbitrary neighbor of $v$ in $T_{v}$. We construct the threshold assignment $\tau_{1}$ for $F_{1}$ as follows.

$$
\tau_{1}(w)= \begin{cases}\varphi(w) & \text { if } w \notin T_{v}, \\ 0 & \text { if } w \in T_{v} \backslash\left\{v, v^{\prime}\right\} \\ \operatorname{deg}_{F_{1}}(w) & \text { if } w \in\left\{v, v^{\prime}\right\}\end{cases}
$$

By Lemma 2, we have $\overline{\tau_{1}} \leq \bar{\varphi}$. Since edge $v v^{\prime}$ is a $\tau_{1}$-resistant subgraph in $F_{1}$, then $\operatorname{dyn}_{\tau_{1}}\left(F_{1}\right)=\operatorname{dyn}_{\varphi}\left(F_{1}\right)=k$ and so $D_{1}$ is a minimum $\tau_{1}$-dynamo of $F_{1}$. Set $F_{2}=F_{1} \backslash T_{v}$. Let $u$ be the parent of the vertex $v$. Construct the threshold assignment $\tau_{2}$ for $F_{2}$ as follows.

$$
\tau_{2}(w)= \begin{cases}\tau_{1}(w) & \text { if } w \in F_{2} \backslash\{u\} \\ \tau_{1}(w)-1 & \text { if } w=u\end{cases}
$$

It is easily seen that the union of any $\tau_{2}$-dynamo of $F_{2}$ and $\{v\}$ is a $\tau_{1}$-dynamo of $F_{1}$ and also $D_{1} \backslash\{v\}$ is a $\tau_{2}$-dynamo of $F_{2}$. Hence $d y n_{\tau_{2}}\left(F_{2}\right)=d y n_{\tau_{1}}\left(F_{1}\right)-1=k-1$. Let $\varphi_{2}$ be any threshold assignment for $F_{2}$ with $\overline{\varphi_{2}}=\overline{\tau_{2}}$. Now construct the threshold assignment $\varphi_{1}$ for $F_{1}$ as follows.

$$
\varphi_{1}(w)= \begin{cases}\varphi_{2}(w) & \text { if } w \in F_{2} \backslash\{u\} \\ \tau_{1}(w) & \text { if } w \in T_{v} \\ \varphi_{2}(w)+1 & \text { if } w=u\end{cases}
$$

Because the union of any $\varphi_{2}$-dynamo of $F_{2}$ and $\{v\}$, forms a $\varphi_{1}$-dynamo of $F_{1}$ and also for any $\varphi_{1}$-dynamo $P$ of $F_{1}$, the set $P \cap F_{2}$ is a $\varphi_{2}$-dynamo of $F_{2}$ then $P \nsubseteq F_{2}$. 
This result and $\operatorname{dyn}_{\tau_{2}}\left(F_{2}\right)=k-1$ imply that $\operatorname{Ldyn}_{\bar{\tau}_{2}}\left(F_{2}\right)=k-1$. From the induction hypothesis there exists a (zero,degree)-assignment $\tau_{2}^{\prime}$ for $F_{2}$ with $\overline{\tau_{2}^{\prime}} \leq \overline{\tau_{2}}$ such that $d y n_{\tau_{2}^{\prime}}\left(F_{2}\right)=k-1$. Now we construct the (zero,degree)-assignment $\tau_{1}^{\prime}$ for $F_{1}$ as follows.

$$
\tau_{1}^{\prime}(w)= \begin{cases}\tau_{2}^{\prime}(w) & \text { if } w \in F_{2} \backslash\{u\} \\ \tau_{1}(w) & \text { if } w \in T_{v} \\ \tau_{2}^{\prime}(w)+1 & \text { if } w=u \text { and } \tau_{2}^{\prime}(u) \neq 0 \\ 0 & \text { if } w=u \text { and } \tau_{2}^{\prime}(u)=0\end{cases}
$$

It is easily seen that $d y n_{\tau_{1}^{\prime}}\left(F_{1}\right)=k$. We finally obtain the desired (zero,degree)assignment $\tau^{\prime}$ for $F$ as follows.

$$
\tau^{\prime}(w)= \begin{cases}\operatorname{deg}_{F}(w) & \text { if } w \in F_{1}, \tau_{1}^{\prime}(w)=\operatorname{deg}_{F \backslash F_{1}}(w), \\ 0 & \text { if } w \in F_{1}, \tau_{1}^{\prime}(w)=0 \\ 0 & \text { if } w \notin F_{1}\end{cases}
$$

In the following we show that for any forest there exists a (zero,degree)-assignment which is zero outside the vertices of a matching.

Proposition 6. Let $F$ be a forest and $t$ a positive constant. Then there exists a matching $M$ such that for the (zero,degree)-assignment $\tau$ defined below, we have $\bar{\tau} \leq t$ and $\operatorname{Ldyn}_{t}(F)=\operatorname{dyn}_{\tau}(F)=|M|$,

$$
\tau(w)= \begin{cases}\operatorname{deg}_{F}(w) & \text { if } w \text { is a vertex saturated by } M \\ 0 & \text { otherwise }\end{cases}
$$

Proof. By Theorem 2, there exists a (zero,degree)-assignment $\tau^{\prime}$ such that $\overline{\tau^{\prime}} \leq t$ and $\operatorname{Ldyn}_{t}(F)=d y n_{\tau^{\prime}}(F)$. Let $F_{1}$ be a subgraph induced on all vertices $w$, with $\tau^{\prime}(w)=\operatorname{deg}_{F}(w)$. Let $D$ be a minimum vertex cover of $F_{1}$. Remark 1 implies that $D$ is a minimum $\tau^{\prime}$-dynamic monopoly of $F$. Assume that $M$ is a maximum matching of $F_{1}$. We show that $M$ satisfies the conditions of the theorem. Each edge of $M$ forms a $\tau$-resistant subgraph in $F$. Hence $d y n_{\tau}(F) \geq|M|$. Using the so-called König Theorem on bipartite graphs we have $|D|=|M|$. Consequently, $\operatorname{dyn}_{\tau}(F) \geq|D|=\operatorname{dyn}_{\tau^{\prime}}(F)=\operatorname{Ldyn}_{t}(F)$. It is easily seen that $\bar{\tau} \leq \overline{\tau^{\prime}} \leq t$. The proof completes.

To prove Theorem [3, we need the following proposition whose proof is given in the appendix.

Proposition 7. Let $G$ be a bipartite graph, where each edge e has a cost $c(e) \geq 0$. Let also $d$ be a positive number. Then there is a polynomial time algorithm which finds a maximum matching $M$ in $G$ with $\operatorname{cost}(M) \leq d$, where $\operatorname{cost}(M)=\sum_{e \in M} c(e)$. 
We are ready now to present the next result.

Theorem 3. Given a forest $F$ and a positive number $t$, there exists an algorithm which computes Ldyn $_{t}(F)$ in polynomial-time.

Proof. For each edge $e=u v$ of $F$ define $\operatorname{cost}(e)=\operatorname{deg}_{F}(u)+\operatorname{deg}_{F}(v)$ and for each $S \subseteq E(F)$ define $\operatorname{cost}(S)=\sum_{e \in S} \operatorname{cost}(e)$. Let $M$ be any arbitrary matching and $\tau$ be a (zero,degree)-assignment constructed from $M$ as obtained in Proposition 6. It is easily seen that $\bar{\tau} \leq t$ if and only if $\operatorname{cost}(M) \leq t|F|$. Now, if $M$ is a maximum matching satisfying $\operatorname{cost}(M) \leq t|F|$, then Proposition 6 implies $\operatorname{Ldyn}_{t}(F)=d y n_{\tau}(F)=|M|$. By Proposition 7 there is a polynomial-time algorithm which finds maximum matching $M$ in $F$ with $\operatorname{cost}(M) \leq c$ for any value $c$. Then using Proposition 6 for given forest $F$ and constant $t$, there is a polynomial time algorithm which finds a (zero,degree)-assignment $\tau$ such that $\operatorname{Ldyn}_{t}(F)=d y n_{\tau}(F)$. From the other side, finding a minimum vertex cover in bipartite graphs is a polynomial-time problem. Therefore using Remark 1 a minimum $\tau$-dynamic monopoly for $F$ can be found in polynomial-time.

For further researches, it would be interesting to obtain other families of graphs for which Ldynamo $(k)$ has polynomial-time solution. Also we don't know yet whether $\operatorname{Ldynamo}(k) \in N P \cup \operatorname{coNP}$. We guess this is not true.

\section{Appendix}

We prove Proposition 7 using the minimum cost flow algorithm. The minimum cost flow problem (MCFP) is as follows (see e.g. [1] for details).

Let $G=(V, E)$ be a directed network with a cost $c(i, j) \geq 0$ for any of its edges $(i, j)$. Also for any edge $(i, j) \in E$ there exists a capacity $u(i, j) \geq 0$. We associate with each vertex $i \in V$ a number $b(i)$ which indicates its source or sink depending on whether $b(i)>0$ or $b(i)<0$. The minimum cost flow problem (MCFP) requires the determination of a flow mapping $f: E \rightarrow \mathbb{R}$ with minimum cost $z(f)=\sum_{(i, j) \in E} c(i, j) f(i, j)$ subject to the following two conditions:

(1) $0 \leq f(i, j) \leq u(i, j)$ for all $(i, j) \in E$ (capacity restriction);

(2) $\sum_{\{j:(i, j) \in E\}} f(i, j)-\sum_{\{j:(j, i) \in E\}} f(j, i)=b(i)$ for all $i \in V$ (demand restriction).

In [1] , a polynomial-time algorithm is given such that determines if such a mapping $f$ exists. And in case of existence, the algorithm outputs $f$. Furthermore, if all values $u(i, j)$ and $b(i)$ are integers then the algorithm obtains an integer-valued mapping $f$. In the following we prove Proposition 7. 
Theorem. Let $G[X, Y]$ be a bipartite graph with $\operatorname{cost}(i j) \geq 0$ for each edge $i j \in G$ and $d$ be a positive number. Then there exists a polynomial-time algorithm which finds maximum matching $M$ in $G$ with $\operatorname{cost}(M) \leq d$.

Proof. Construct a directed network $H$ from bipartite graph $G[X, Y]$ as follows. Add two new vertices $s$ and $t$ as the source and the sink of $H$, respectively and directed edges $(s, x)$ for each $x \in X$ and $(y, t)$ for each $y \in Y$. Make all other edges directed from $X$ to $Y$. For each edge $(i, j)$ set $u(i, j)=1$ and define $c(i, j)$ as follows.

$$
c(i, j)= \begin{cases}0 & i=s \text { or } j=t, \\ \operatorname{cost}(i j) & i \in X, j \in Y .\end{cases}
$$

For each vertex $i \in X \cup Y$, set $b(i)=0$ and define $b(s)=-b(t)=k$, where $k$ is an arbitrary positive integer. We have now an instance of MCFP. Assume that there exists a minimum cost flow mapping for this instance (obtained by the above-mentioned algorithm of [1]). Since $u(i, j)$ and $b(i)$ are integers then $f$ is an integer-valued mapping. Therefore $f(i, j)$ is either 0 or 1 . Let $M$ be the set of edges $(i, j)$ with $f(i, j)=1$, where $i \in X$ and $j \in Y$. Clearly $M$ is a matching of size $k$ having $\operatorname{cost}(M)=z(f)$, where $z(f)$ is as defined in MCFP above.

Conversely, let $M^{\prime}$ be any arbitrary matching in $G$ with $\left|M^{\prime}\right|=k$. We construct a flow mapping $f$ as follows.

$$
f(i, j)= \begin{cases}1 & i \in X, j \in Y, i j \in M^{\prime} \\ 1 & i=s, j l \in M^{\prime} \text { for some } l \in Y, \\ 1 & j=t, l i \in M^{\prime} \text { for some } l \in X \\ 0 & \text { otherwise }\end{cases}
$$

The conditions of MCFP are satisfied for $f$. Also $z(f)=\operatorname{cost}\left(M^{\prime}\right)$. We conclude that to obtain a matching of size $k$ with the minimum cost is equivalent to obtain a minimum cost flow mapping for the associated MCFP instance (note that $k$ is a parameter of this instance). We conclude that in order to find a matching $M$ satisfying $\operatorname{cost}(M) \leq d$ and with the maximum size, it is enough to run the corresponding algorithm for the above-constructed MCFP instance for each $k$, where $k$ varies from 1 to $|G| / 2$. Note that $|G| / 2$ is an upper bound for the size of any matching. This completes the proof.

\section{References}

[1] R.K. Ahuja, T.L. Magnanti, J.B. Orlin, Network flows: theory, algorithms, and applications, Prentice Hall 1993. 
[2] J.A. Bondy, U.S.R. Murty, Graph Theory, Springer 2008.

[3] C-L. Chang, Y-D. Lyuu, On irreversible dynamic monopolies in general graphs, arXiv:0904.2306v3, 2010.

[4] C.C. Centeno, M.C. Dourado, L.D. Penso, D. Rautenbach , J.L. Szwarcfiter, Irreversible conversion of graphs, Theoret. Comput. Sci. 412 (2011) 3693-3700.

[5] C.C. Centeno, D. Rautenbach, Remarks on dynamic monopolies with average thresholds, manuscript 2013.

[6] N. Chen, On the approximability of influence in social networks, SIAM J. Discrete Math. 23 (2009) 1400-1415.

[7] P.A. Dreyer, F.S. Roberts, Irreversible $k$-threshold processes: Graph-theoretical threshold models of the spread of disease and of opinion, Disc. Appl. Math. 157 (2009) 1615-1627.

[8] P. Flocchini, R. Kralovic, A. Roncato, P. Ruzicka, N. Santoro, On time versus size for monotone dynamic monopolies in regular topologies, J. Discrete Algorithms, 1 (2003) 129-150.

[9] M.R. Garey, D.S. Johnson, Computers and Intractability: A Guide to the Theory of NP-Completeness, W. H. Freeman \& Co., New York, NY, USA, 1979.

[10] K. Khoshkhah, H. Soltani, M. Zaker, On dynamic monopolies of graphs: The average and strict majority thresholds, Discrete Optim. 9 (2012) 77-83.

[11] H. Soltani, M. Zaker, Dynamic monopolies of graphs with probabilistic thresholds, submitted.

[12] M. Zaker, On dynamic monopolies of graphs with general thresholds, Disc. Math., 312 (2012) 1136-1143.

[13] M. Zaker, Generalized degeneracy, dynamic monopolies and maximum degenerate subgraphss, Disc. Appl. Math., 161 (2013) 2716-2723. 